\title{
Spirituality moderates hopelessness, depression, and suicidal behavior among Malaysian adolescents
}

\begin{abstract}
Suicide is an important public health problem for adolescents, and it is essential to increase our knowledge concerning the etiology of suicide among adolescent students. Therefore, this study was designed to examine the associations between hopelessness, depression, spirituality, and suicidal behavior, and to examine spirituality as a moderator between hopelessness, depression, and suicidal behavior among 1376 Malaysian adolescent students. The participants completed measures of depression, hopelessness, daily spiritual experience, and suicidal behavior. Structural equation modeling indicated that adolescent students high in hopelessness and depression, but also high in spirituality, had less suicidal behavior than others. These findings reinforce the importance of spirituality as a protective factor against hopelessness, depression, and suicidal behavior among Malaysian adolescent students.
\end{abstract}

Keyword: Feedback control; Binary fluid; Soret parameter; Dufour 
